\title{
The luminance of tachistoscope lamps as a function of flash duration
}

\author{
ROBERT W. BOHLANDER \\ University of Rochester, Rochester, New York 14627
}

\begin{abstract}
The steady-state luminance of the lamps in a commonly used Iconix tachistoscope was shown to depend, in a complex nonlinear fashion, upon the length of time the lamps were lit. This was probably due to variations in lamp temperature with time. An integrating photometer was used to measure the light energy present in short flashes and in time periods of various durations with the lamps burning continuously. The equivalent luminance of short flashes was calculated by comparing values obtained by integration over a short flash with values resulting from integration, over an identical duration, of the lamps in a steady-state. With flashes up to $50 \mathrm{msec}$ in nominal duration, luminous energy was less than one-half of that present in the same duration with the lamps burning continuously. The importance of this information for the interpretation of tachistoscopic experiments, as well as possible means of monitoring the effects, are discussed.
\end{abstract}

The tachistoscope (T-scope) has proved to be an extremely useful tool in many areas of perceptual research. Numerous experiments have utilized the apparatus for studying such phenomena as visual reactiontime, masking, and luminance thresholds. In these studies, the luminance levels of fixation and stimulus fields are typically reported in some detail. Usually the data are obtained through photometric measurement of the "steady-state" luminance of the T-scope field, with the lamps burning continuously. In most experiments, however, the lamps are not burned continuously: they are flashed briefly. Experimenters generally assume that the luminance of a briefly flashed field (typically a fraction of a second) is equivalent to its steady-state luminance and that the luminance remains fairly constant across experimental trials. These assumptions are vital to interpretations of reaction-time data collected with a T-scope, since stimulus luminance is critical in reactiontime studies (Payne, 1967; Rains, 1963; Teichner \& Krebs, 1974), especially at the low luminances obtainable with multichannel T-scopes.

Siegel (1968) recognized the importance of the above assumptions and examined some of the luminance characteristics of T-scope lamps as a function of on-time. His findings indicated that the temperature of the phosphor-coated lamps used in T-scopes was an important factor in determining light output. Generally, the warmer the lamp, the greater its output. For this reason, the amount of time a lamp burned prior to a given experimental trial affected the luminance of the stimulus field. Siegel found that a lamp which had warmed up for $15 \mathrm{~min}$ or more tended to stabilize in luminance; brief flashes (up to $20 \mathrm{msec}$ ) never reached stability.

More recently, Mollon and Polden (1978) examined the time constants of several commercially available $\mathrm{T}$-scopes and found them to be considerably longer than claimed by the manufacturers. Typically, $18 \mathrm{msec}$ was the rise time for the light output to reach $90 \%$ of its peak value and the decay time for the light output to fall to $10 \%$ of its peak. Under certain conditions, visible light was present up to $40 \mathrm{msec}$ after the nominal offset of the lamps.

The present study was undertaken with three goals in mind: (1) to discover the types of temporal-luminance interactions existing in a commonly used brand of T-scope, (2) to obtain more specific data than that reported by Siegel (1968) in order to assess the magnitude of the luminance fluctuations that exist in T-scopes (such information permits a reevaluation of previous experiments in which the luminance levels of the T-scopes are critical), and (3) to examine a possible means of measuring the luminance of very short flashes of light and of monitoring luminance during an experiment.

\section{APPARATUS}

All studies to be reported utilized the following Iconix T-scope components (Siliconix, Inc.): fourfield tachistoscope exposure box, Model 61374; programmable time-base generator and counter, Model 6255; preset controller, Model 6010; four-channel tachistoscope lamp driver, Model 6192-4. The lamps used in each channel of the system were pairs of 6-W F6T5/CW fluorescent tubes (Westinghouse Corporation).

\section{STEADY-STATE LUMINANCE AS A FUNCTION OF ON-TIME}

The luminances of three fields of the T-scope were monitored periodically from $10 \mathrm{sec}$ to $40 \mathrm{~min}$ after the lamps were turned on. All measurements were made 
with a digital photometer (Tektronix, Inc.) positioned at the viewing port, with opaque white cards placed in the stimulus card holders. Values were recorded every $10 \mathrm{sec}$ for the first $3 \mathrm{~min}$, every minute for the next $7 \mathrm{~min}$, and every $5 \mathrm{~min}$ thereafter. The luminances of all three channels exhibited similar fluctuations over time, and the mean data appear in Figure 1. The output of the three pairs of lamps increased rapidly after turn-on, peaked at about $2 \mathrm{~min}$, and then gradually decreased over an extended period of time. The peak luminance was at a level approximately $60 \%$ above the initial reading for each channel, whereas the fall-off after the peak was only $6 \%$.

According to the manufacturer, luminance is proportional to the current driving the lamps. Since it was possible that changes in current over time might be responsible for the observed luminance variations, current from the lamp driver was monitored during these trials with a digital multimeter (Keithley Instruments, Inc.). Only minor, random fluctuations of current (less than $.5 \%$ ) occurred. Since the luminance changes observed during the same period were about $60 \%$ or greater, some characteristics of the lamps themselves must have caused the luminance variation.

The present results differ somewhat from those of Siegel (1968), who found that luminance increased with time after turn-on, then became stable after 10-15 min. Since Siegel did not specify the apparatus tested, the reason for this discrepancy remains unclear.

According to the Lighting Handbook (Westinghouse Electric Corporation, 1969), the light output of hot cathode tubes is at a peak at $80^{\circ} \mathrm{F}$ but falls off as lamp temperature increases or decreases. The present results may easily be explained on this basis if it is assumed that, after the lamps were turned on at room temperature

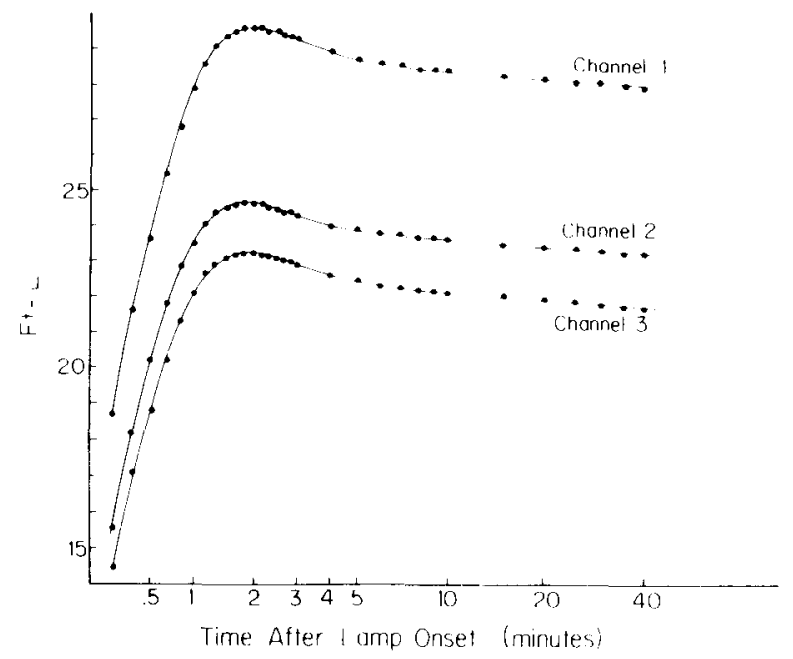

Figure 1. Luminance of three $T$-scope channels as a function of lamp on-time. Data points are means of three trials for each channel. Abscissa is a logarithmic scale. Lamp-driver intensity is set at a maximum for all channels; vertical displacement of curves represents normal interchannel luminance variability. $\left(70^{\circ} \mathrm{F}\right)$, their temperature gradually increased and passed $80^{\circ} \mathrm{F}$, the point of peak output.

\section{MONITORING LUMINANCE WITH A SOLAR CELL AND OSCILLOSCOPE}

Because all three channels showed similar changes, a single channel (Channel 3) was chosen for more extensive examination. A silicon photovoltaic cell (solar cell) was placed into the channel, facing the stimulus card. Its voltage output was monitored on a storage oscilloscope.

Silicon cells of this type are maximally sensitive to infrared radiation in the region of $850-900 \mathrm{~nm}$. Their short-wavelength cutoff of about $300 \mathrm{~nm}$ permits detection of near ultraviolet wavelengths. Mollon and Polden (1978) indicated that ultraviolet and infrared radiation were present in the output of T-scope lamps. This information was considered in the present study. To eliminate the invisible radiation from the measurements made with the solar cell, a CIE photopic correction filter (P-102, Optronics, Inc.) was positioned over the cell.

Luminance measurements were made concurrently with the photometer and the solar cell. After the lamps had been on for $1 \mathrm{~h}$, the voltage output of the solar cell was calibrated against the photometer readings; as the current driving the lamps was continuously varied over the entire range possible (about $20 \mathrm{fL}$ ). The output of the solar cell varied linearly with the logarithm of the luminance, and a regression equation was determined. The use of the solar cell technique, and knowledge of the equation, permits unobtrusive monitoring of luminance during the course of an experiment.

\section{HOW MUCH LIGHT ENERGY IS PRESENT IN BRIEF FLASHES?}

The T-scope's primary utility is in presenting brief flashes of light, so it is important to know how luminance varies with respect to flash duration. Flashes of three durations were examined, and the profiles of a 1-, 10-, and 20-msec flash, traced from the oscilloscope screen, appear in Figure 2. The lamps approached a peak output in about $20 \mathrm{msec}$, which agrees with the Mollon and Polden (1978) findings for that type of lamp. Also in accord with their results is the slow decay time evident in Figure 2.

If one considers the data in Figure 1, it is evident that the "peak" output of the T-scope lamps, approached with a 20 -msec flash, is likely to be far below the peak obtainable with the lamps burning continuously. About $2 \mathrm{~min}$ are required for the lamps to reach a steady-state peak that is $60 \%$ above their initial luminance level. The 1-, 10-, and 20-msec flashes presented in Figure 2, with the lamps cold prior to each flash, correspond to the very earliest stages of the curves in Figure 1. For this reason, light output would be expected to fall far short of the steady-state peak. 


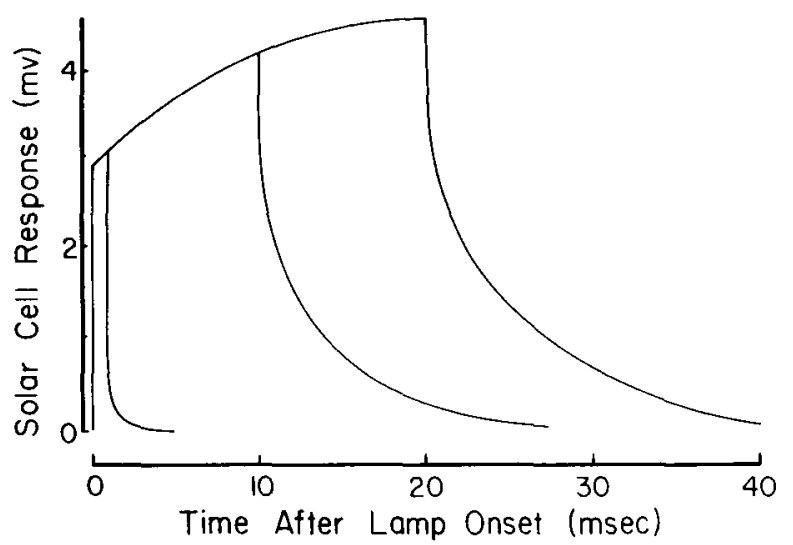
flash.

Figure 2. Solar cell responses to a 1-, 10, and 20-msec

As flash duration increases and the lamps become warmer, one expects the peak light output achieved by the lamps to increase also. Figure 3 shows the oscilloscope traces of four flashes of different durations, with the time scale adjusted so that they are superimposed. Increasing flash duration did result in higher peak light output. Likewise, two flashes of the same duration, but occurring under differing conditions of lamp temperature, exhibited differences in light output. Figure 4 shows an example of the effect that lamp temperature had on the light output of $100-\mathrm{msec}$ flashes. The warmer lamp not only reached a peak output above that of the cold lamp, but also decayed more slowly.

Due to the important effects of lamp temperature, the luminous energy present in brief flashes used during an experiment may be much lower than values determined photometrically with the lamps on constantly. A precise way to measure the amount of light present in a short flash is through the use of an integrating photometer. In the present study, an Optronics radiometer/photometer (Model 730A) was used. The fiber optic probe, fitted with the CIE photopic correction filter (previously used in conjunction with the solar cell), was placed in the viewing port of the T-scope. ${ }^{1}$

With the photometer set in the integrating mode, the integrating time gate, or the span over which integration of light energy occurs, could be varied continuously from 5 to $70 \mathrm{msec}$. Three gates were utilized: 10,30 , and $50 \mathrm{msec}$. Before integrating the energy in brief flashes of light, the steady-state condition was examined with the lamps burning continuously. The luminance of the T-scope was varied over its possible range and, at each 1-fL interval, the light energy present in 10-, 30-, and 50 -msec time bins was recorded. The mean data for 10 trials at each of the three time gates appear in Figure 5, along with the regression lines calculated for each gate.

Next, with the steady-state luminance preset at $20 \mathrm{fL}$, the energy in light flashes of durations equal to each of the three time gates was integrated. One milli- second before each flash, a signal from the lamp driver triggered the photometer to begin integration. ${ }^{2}$ The values of light energy obtained in this manner were compared with the corresponding time gate curve in Figure 5. This permitted a determination of an "equivalent" luminance value in terms of foot Lamberts.

For example, integration of a $50-\mathrm{msec}$ flash, over a $50-\mathrm{msec}$ time period, yielded a reading of $.194 \times 1 \sigma^{6}$ lumens $(\mathrm{sec}) / \mathrm{cm}^{2}$. When this value was compared to the curve in Figure 5 for a 50 -msec integration of steadystate light, a similar value resulted from a $50-\mathrm{msec}$ integration of a continuously burning lamp whose luminance was about $7 \mathrm{fL}$. Thus a $50-\mathrm{msec}$ flash of (nominally) $20 \mathrm{fL}$ contained luminous energy equivalent to $50 \mathrm{msec}$ of a $7 . \mathrm{fL}$ light buming continuously. Even when the time gates used to integrate the flashes were extended $20 \mathrm{msec}$ beyond the nominal flash dura-

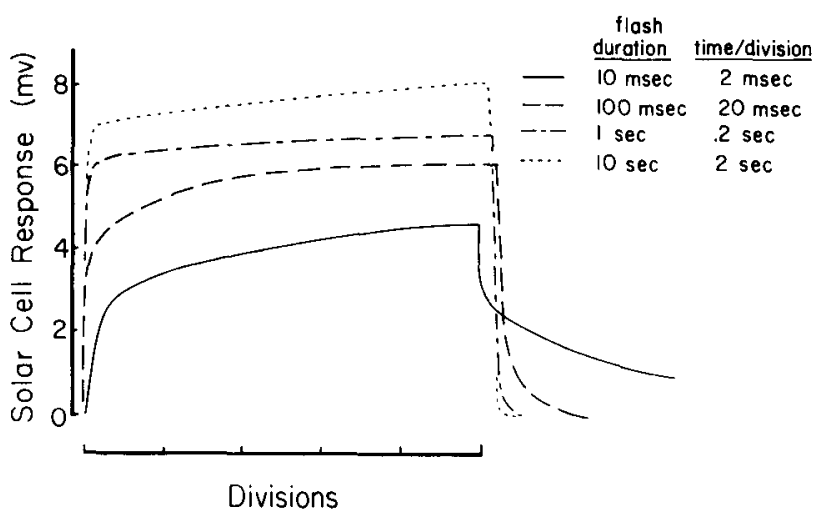

Figure 3. Solar cell responses to flashes of four durations. Oscilloscope voltage scale was constant at $1 \mathrm{mV} /$ division for all traces.

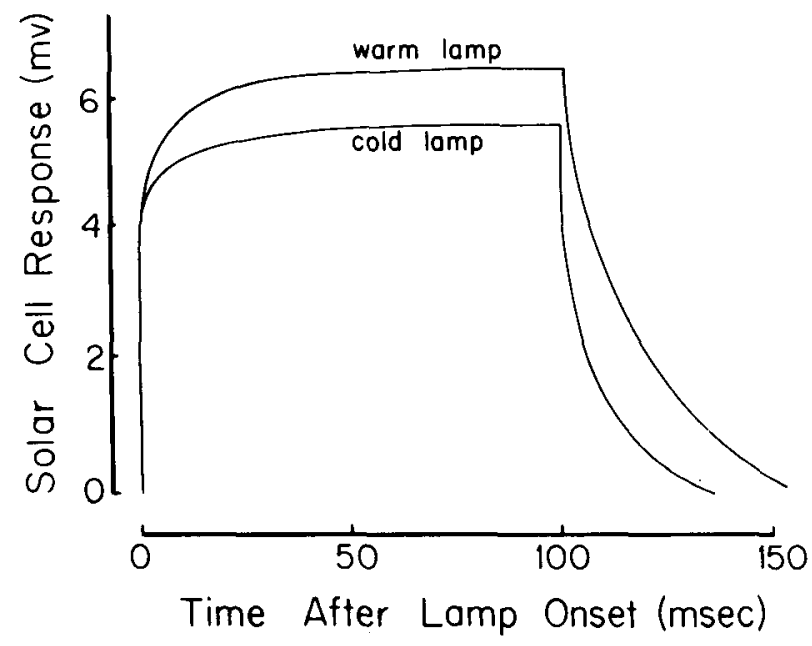

Figure 4. Solar cell responses to a 100-msec flash from a cold lamp and a 100-msec flash from a warm lamp. "Warm" condition is defined as a lamp lit for $1 \mathrm{~min}$ prior to the flash; "cold" indicates the lamp is at room temperature. 


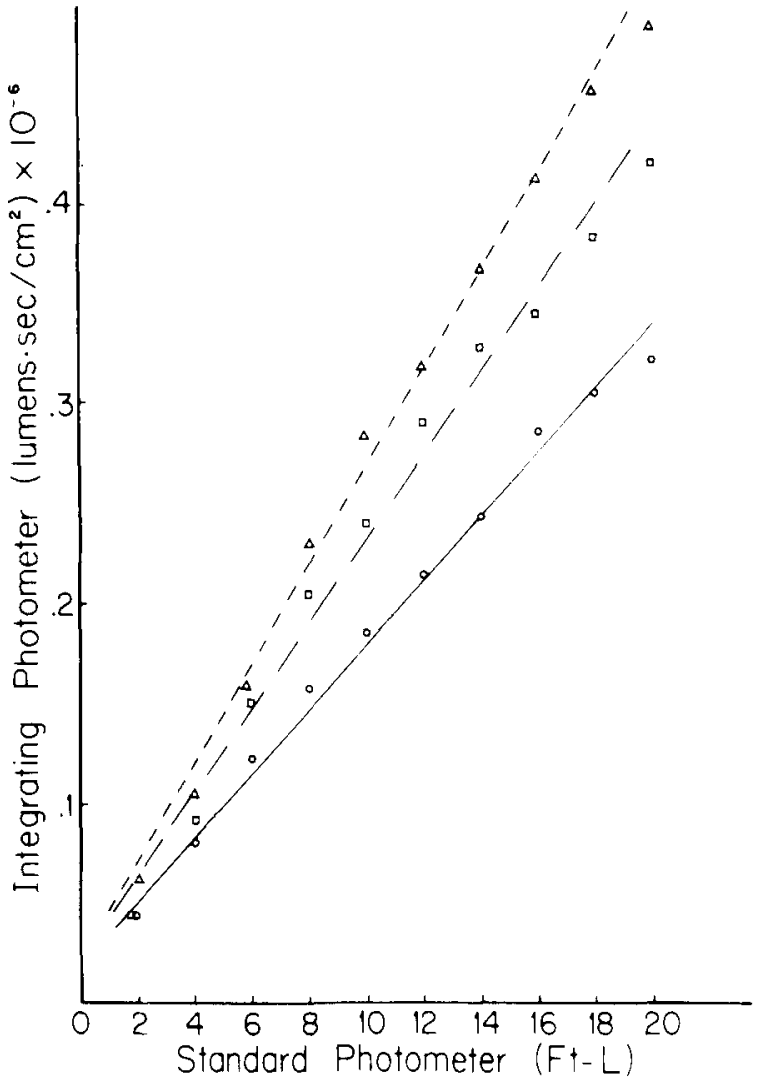

Figure 5. Mean luminous energy emitted by the T-scope lamps as steady-state luminance is varied. Symbols: $\Delta, 50-\mathrm{msec}$ integration; $\square, 30$ msec integration; $O, 10$-msec integration. Regression lines: $(\cdots), 50-m s e c$ data; $(--), 30-m s e c$ data; (-), 10-msec data.

tion, to account for energy present during the decay phase of the flashes, the total amount of energy was still far below the steady-state value.

All determinations of luminous energy were repeated under two conditions of lamp temperature: one in which the lamps were allowed to cool to room temperature before each trial, the other in which lamps were warmed by burning for 1 min prior to each trial.

Figure 6 shows the means of 20 determinations for: (1) each of three flash durations, (2) time gates equal to, and $20 \mathrm{msec}$ longer than, flash duration, and (3) warm and cold lamps. As flash duration increased, the equivalent number of foot Lamberts approached the nominal steady-state value. Even with a 50 -msec flash integrated over $70 \mathrm{msec}$, the total luminous energy was less than one-half the steady-state value. Lamp temperature also affected light output, with warmer lamps yielding amounts of energy about $70 \%$ above values for cold lamps.

\section{CONCLUSIONS}

The principal conclusion of this study is that experimenters who utilize standard photometric measurements to specify the luminance of short flashes of light may be overestimating the output of their T-scope lamps. The large increase in light output with lamp temperature (about $60 \%$ ), and the length of time required for the lamps to reach steady state $(2 \mathrm{~min})$, result in flashes whose luminous energy is much less than expected on the basis of steady-state measurements. The luminance of flashes up to $50 \mathrm{msec}$ in nominal duration, under the conditions tested here, was less than half that measured with the lamps burning continuously. This finding suggests the possibility that conclusions drawn in previous studies with T-scopes, in which luminance was a critical factor, are in error. A reevaluation of these studies is in order, as alternative interpretations of the data collected may now be possible.

The present study supports the general conclusion of Mollen and Polden (1978) concerning the long time constants of T-scope lamps. The examination of the waveforms of $\mathrm{T}$-scope flashes tends to be misleading, however. The peaks of short flashes represent only a fraction of the light output obtainable if the lamps are permitted to remain on for several minutes. The peaks of flash waveforms increased steadily with flash duration.

Mollon and Polden state that "Experiments that have varied exposure duration in the range of 0 to $50 \mathrm{~ms}$ have also unintentionally varied stimulus intensity." Present findings indicate that the range of flash durations can be extended to $2 \mathrm{~min}$.

Generalization of these findings to other types of T-scope lamps must await further testing. Similarities in the construction of $\mathrm{T}$-scope lamps, however, suggest that for many types of lamps temperature probably plays some role in determining light output.

It would be advantageous for individual investigators to attempt to control for the variable of lamp temperature, and hence maintain a more stable luminance level, by keeping a strict schedule of on-and off-times for the lamps. If additional steps are taken, such as those

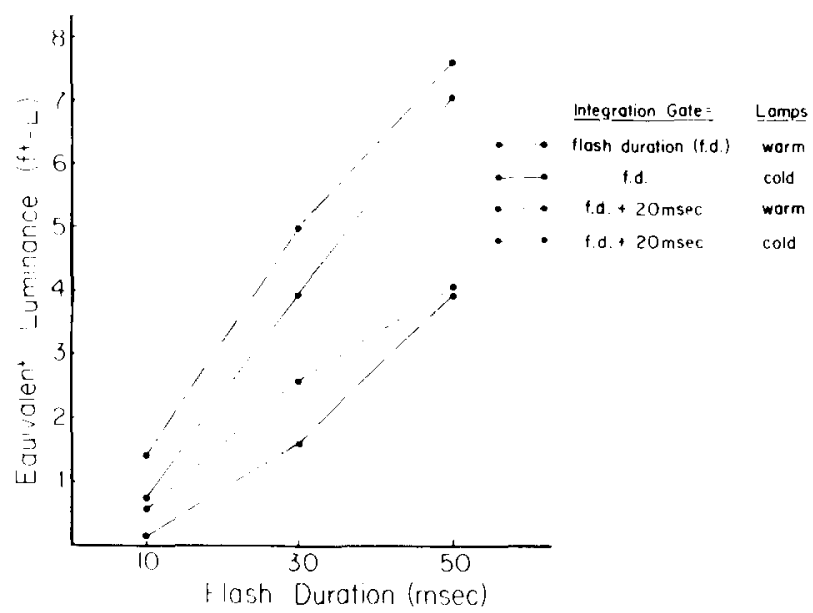

Figure 6. Equivalent luminance of brief flashes as a function of their duration. Values were obtained by comparison of integration data with appropriate curves of Figure 5. Steadystate luminance was held constant at $20 \mathrm{fL}$. 
employed here, the rise and decay time, peak luminance level, and equivalent luminance can be determined. These data should provide the investigator with adequate information about the luminance characteristics of the specific apparatus being utilized.

\section{REFERENCES}

Mollon, J. D., \& Polden, P. G. On the time constants of tachistoscopes. Quarterly Journal of Experimental Psychology, 1978, 30, 555-568.

PAyne, W. H. Visual reaction time on a circle about the fovea. Science, 1967, 155, 481-482.

RaINS, J. D. Signal luminance and position effects in human reaction time. Vision Research, 1963, 3, 239-251.

Siegel, M. H. A note on luminance control in tachistoscopes. Behavior Research Methods \& Instrumentation, 1968, 1, 33.

TeichneR, W. H., \& KREBS, M. J. Laws of visual choice reaction time. Psychological Review, 1974, 81, 75-98.

Westinghouse Electric Corporation. Lighting handbook. Bloomfield, N.J: Westinghouse Electric, 1969.

\section{NOTES}

1. Due to physical restraints, the probe could not be placed at the exact position previously occupied by the standard photometer. Therefore, the integrating photometer (set in its nonintegrating mode for use as a simple photometer) was calibrated against the standard photometer used earlier. The crosscalibration yielded a nearly perfect linear relationship between the number of foot Lamberts read from the standard photometer and the unit setting on the integrating photometer [lumens (seconds) $/ \mathrm{cm}^{2}$ ]. All readings from the integrating photometer could now be directly converted into foot Lamberts for easy comparison with earlier data.

2. This procedure, recommended by the manufacturer of the integrating photometer, guarantees that the appropriate relays within the photometer have operated by the time the light flash is initiated.

(Received for publication February 27, 1979; revision accepted May $17,1979$. 